\title{
DEGRADAÇÃO TÉRMICA DE RESÍDUOS DE MEDIUM DENSITY FIBERBOARD (MDF): ESTUDO DA CINÉTICA DA REAÇÃO DE PIRÓLISE
}

\author{
S. D. FERREIRA ${ }^{1}$, C. R. ALTAFINI ${ }^{1}$, D. PERONDI ${ }^{2}$, M. GODINHO ${ }^{3}$, P. $^{2}$ \\ MARCOLIN $^{3}$ \\ ${ }^{1}$ Universidade de Caxias do Sul, Programa de Pós-Graduação em Engenharia Mecânica \\ ${ }^{2}$ Universidade Federal do Rio Grande do Sul, Programa de Pós-Graduação em \\ Engenharia de Minas, Metalúrgica e de Materiais \\ ${ }^{3}$ Universidade de Caxias do Sul, Programa de Pós-Graduação em Engenharia de \\ Processos e Tecnologias \\ ${ }^{4}$ Universidade de Caxias do Sul, Departamento de Engenharia Química \\ E-mail para contato: suelemferreira2006@yahoo.com.br

\begin{abstract}
RESUMO - Resíduos de fibra de média densidade (MDF) são gerados pelo setor moveleiro. $\mathrm{O}$ estudo da cinética da pirólise do MDF é importante para a compreensão do mecanismo controlador da reação, e é necessário para a simulação do processo de pirólise. A cinética da degradação térmica de resíduos de MDF foi avaliada em condições não-isotérmicas através de análises termogravimétricas (TGA/DTG) em atmosfera inerte $\left(\mathrm{N}_{2}\right)$. Os experimentos foram realizados com amostras de diâmetro inferior a $0,21 \mathrm{~mm}$ e massa inicial de aproximadamente $10 \mathrm{mg}$. Para os ensaios foram utilizadas as seguintes taxas de aquecimento: $5,10,15,25$ e 50,75 e $100{ }^{\circ} \mathrm{C} / \mathrm{min}$. O triplete cinético foi determinado através dos métodos não-isoconvencionais (Coats-Redfern/ MacCallum-Tanner/ Van Krevelen). Os experimentos em atmosfera inerte mostraram que a região onde ocorre a principal perda de massa do MDF ocorre entre $200{ }^{\circ} \mathrm{C}$ e $500{ }^{\circ} \mathrm{C}$, a energia de ativação variou de 137 a $152 \mathrm{~kJ}^{\circ} \mathrm{mol}^{-1}$ e o fator pré-exponencial de $2 \cdot 10^{10}$ a $5 \cdot 10^{11} \mathrm{~min}^{-1}$.
\end{abstract}

\section{INTRODUÇÃO}

Atualmente há uma grande preocupação em substituir os combustíveis fósseis por fontes limpas de energia, devido às emissões de gases poluentes na atmosfera, resultando em políticas que visam substituir esses combustíveis por combustíveis menos agressivos, como é a caso da biomassa (MUNIR et al., 2009).

O MDF no Brasil é muito utilizado pelas indústrias de móveis devido à sua estrutura que garante resultados satisfatórios no uso de técnicas convencionais. No MDF as fibras de madeira são aglutinadas e compactadas entre si com resina sintética através da ação conjunta de pressão e calor. Segundo a Associação Brasileira da Indústria de Painéis de Madeira (ABIPA, 2010), os painéis são produzidos com madeiras provenientes de florestas plantadas dos gêneros Pinus e Eucalyptus.

Em sua composição o MDF possui adição de resinas, em especial a uréiaformaldeído. O percentual aproximado desta resina é de 10\% (Gan et al., 2004). As tecnologias baseadas na pirólise da biomassa exigem um conhecimento da cinética de 
degradação térmica, sendo a mesma uma ferramenta útil, para modelar e avaliar os melhores métodos de trabalho em reatores, fornos, caldeiras ou processos industriais (Hagedorn et al.,2003; Regland \& Aerts,1996).

O estudo da cinética da reação de pirólise é um fator determinante para a definição dos parâmetros cinéticos, como a energia de ativação $\left(\mathrm{E}_{\mathrm{a}}\right)$ e fator préexponencial (A), bem como para determinação do mecanismo da reação. As análises térmicas (TGA/DTG) são amplamente utilizadas para a obtenção destes parâmetros. Sendo que a " $E_{a}$ " é essencialmente sensível a temperatura de reação, enquanto o fator "A" está relacionado com a estrutura do material. Portanto, a reatividade da madeira é caracterizada principalmente pela energia de ativação (ELLA et al., 2005).

Na literatura há diversos estudos envolvendo análises térmicas e determinação dos parâmetros cinéticos para diferentes biomassas. Bianchi et al. (2010), realizaram um estudo termogravimétrico em atmosfera inerte para dois tipos de maneira: pinus e garapeira. Eles estimaram os parâmetros cinéticos pelo método proposto por FlynnWall-Ozawa. Outros trabalhos foram realizados por Munir et al. (2009), Wang et al.; (2006), Guetti, et al. (1996), contudo, ainda são poucos os estudos referentes ao comportamento térmico em especial, do MDF, no processo de pirólise. Os resultados dos estudos referentes aos parâmetros cinéticos para biomassa encontrados na literatura dependem diretamente das condições experimentais, como taxa de aquecimento, tamanho da partícula, entre outros, podendo assim variar os parâmetros obtidos.

A contribuição deste trabalho está no estudo cinético da degradação térmica em atmosfera inerte do MDF, através da determinação do triplete cinético da reação de pirólise.

\section{MATERIAIS E MÉTODOS}

As análises térmicas (TGA/DTG) foram realizadas numa termobalança da marca Shimadzu, modelo TGA-50. Os experimentos foram conduzidos em atmosfera inerte $\left(\mathrm{N}_{2}\right)$ com fluxo de $50 \mathrm{ml} \cdot \mathrm{min}^{-1}$ e nas seguintes taxas de aquecimento: 5, 10, 15, 25 e 50, 75 e $100^{\circ} \mathrm{C} / \mathrm{min}$. Todos os experimentos foram realizados com massa inicial de aproximadamente $10 \mathrm{mg}$, conduzidos da temperatura ambiente até $800{ }^{\circ} \mathrm{C}$ e com amostras de diâmetro inferior a 0,21 mm (mesh tyler70).

Os métodos de ajuste utilizados neste trabalho para definição dos parâmetros cinéticos e do mecanismo controlador para o MDF são os modelos matemáticos de Coats-Redfern, MacCallum-Tanner e van Krevelen. Estes modelos têm sido amplamente utilizados para fornecer informações sobre o mecanismo controlador da cinética (REGNIER \& GUIB, 2007; FRAGA \& NUNEZ, 2001).

Na termogravimetria não-isotérmica a fração de material pirolisado $(\alpha)$ pode ser obtida a partir da equação (1):

$$
\alpha=\frac{m_{i}-m_{\tau}}{m_{i}-m_{f}}
$$

Onde, $\mathrm{m}_{\mathrm{i}}$ é a massa inicial, $\mathrm{m}_{\tau}$ é a massa em qualquer instante a uma temperatura $T$ e $\mathrm{m}_{\mathrm{f}}$ é a massa final do experimento, respectivamente. 
De acordo com (Regnier \& Guibe, 1997; Lua \& Su, 2006; Aboulkas et al., 2009; Jiang et al., 2010 e Perondi, et al., 2012), o processo de pirólise pode ser assumido, conforme a equação (2) e (3):

$$
\frac{d \alpha}{d t}=k(T) \cdot f(\alpha)=A \cdot \exp \left(-\frac{E_{a}}{R T}\right) \cdot f(\alpha)
$$

Onde, $\mathrm{k}(\mathrm{T})$ é a constante da taxa de reação, A é o fator pré-exponencial, $\mathrm{E}_{\mathrm{a}}$ é a energia de ativação aparente, $\mathrm{T}$ é a temperatura absoluta e $\mathrm{R}$ é a constante dos gases. A Eq.(2) é também usada na sua forma integral, para condições não isotérmicas conforme a equação (3):

$$
g(\alpha)=\int_{0}^{\alpha} \frac{d \alpha}{f(\alpha)}=\frac{A}{\beta} \int_{T_{0}}^{T} \exp \left(-\frac{E_{a}}{R T}\right) d T
$$

Onde, $\beta$ é a taxa de aquecimento $(\beta=d T / d t)$.

Na Tabela 1 estão representados os mecanismos avaliados neste trabalho.

Tabela 1 - Modelos matemáticos para $\mathrm{g}(\alpha)$ e $\mathrm{f}(\alpha)$ frequentemente utilizados em reações

\begin{tabular}{|c|c|c|c|}
\hline & Mecanismo & $f(\alpha)$ & $g(\alpha)$ \\
\hline \multirow{3}{*}{ Reação química } & Primeira ordem $(F 1)$ & $(1-\alpha)$ & $-\ln (1-\alpha)$ \\
\hline & Segunda ordem (F2) & $(1-\alpha)^{2}$ & $(1-\alpha)^{-1}-1$ \\
\hline & Ordem n (Fn) & $(1-\alpha)^{n}$ & $\frac{(1-\alpha)^{1-n}-1}{n-1}$ \\
\hline \multirow{2}{*}{$\begin{array}{l}\text { Nucleaçãa- } \\
\text { crescimento }\end{array}$} & Avrami-Erofeev (A2) & $2(1-\alpha)[-\ln (1-\alpha)]^{0,5}$ & {$[-\ln (1-\alpha)]^{0,5}$} \\
\hline & Avrami-Erofeev (A3) & $3(1-\alpha)[-\ln (1-\alpha)]^{0,667}$ & {$[-\ln (1-\alpha)]^{0,333}$} \\
\hline \multirow{4}{*}{ Difusão } & Unidimensional (D1) & $2 \alpha^{-1}$ & $\alpha^{2}$ \\
\hline & Eq. de Valensi (D2) & $-[\ln (1-\alpha)]^{-1}$ & $(1-\alpha) \ln (1-\alpha)+\alpha$ \\
\hline & Eq. de Jander (D3) & $1,5(1-\alpha)^{0,667}\left[1-(1-\alpha)^{0,333}\right]^{-1}$ & {$\left[1-(1-\alpha)^{0,333}\right]^{2}$} \\
\hline & $\begin{array}{l}\text { Eq. De Ginstling- } \\
\text { Brounshtein (D4) }\end{array}$ & $1,5-\left((1-\alpha)^{-0,667}-1\right)^{-1}$ & $1-0,667 \alpha-(1-\alpha)^{0,667}$ \\
\hline \multirow{2}{*}{$\begin{array}{l}\text { Phase Boundary } \\
\text { reaction }\end{array}$} & $\begin{array}{c}\text { Contracting Sphere } \\
(\mathrm{R} 2)\end{array}$ & $2(1-\alpha)^{0,5}$ & {$[1-(1-\alpha)]^{0,5}$} \\
\hline & $\begin{array}{c}\text { Contracting Cylinder } \\
(\mathrm{R} 3)\end{array}$ & $3(1-\alpha)^{0,667}$ & {$[1-(1-\alpha)]^{0,333}$} \\
\hline
\end{tabular}
cinéticas no estado sólido

Fonte: adaptada de (MONTSERRAT et al.,1998; GAO et al.,2002; KIM \& KIM,2005; VLAEV et al.,2008; LUA \& SU,2006; ABOULKA et al.,2009; JIANG et. al.,2010)

O método de Coats-Redfern (COATS \& REDFERN, 1964) é definido segundo a equação 4: 


$$
\ln \left(\frac{g(\alpha)}{T^{2}}\right)=\ln \left[\frac{A R}{\beta E_{a}}\left(1-\frac{2 R T_{\max }}{E_{a}}\right)\right]-\frac{E_{a}}{R T}
$$

Onde, $\mathrm{T}_{\max }$ é a temperatura onde a taxa de reação é máxima. Os parâmetros cinéticos Ea e A são obtidos plotando o gráfico $\ln \left(g(\alpha) / T^{2}\right)$ x $1 / T$, e o resultado é uma linha reta, cuja inclinação e a interseção fornece os respectivos valores.

A função $g(\alpha)$ é a integral da função de conversão, a qual depende do mecanismo que controla a reação e ainda do tamanho e da forma das partículas reagentes.

No método de MacCallum \& Tanner (1970) é utilizada a equação 5.

$$
\log g(\alpha)=\log \frac{A E_{a}}{\beta R}-0,4828 E_{a}^{0,4351}-\left(\frac{0,449+0,217 E_{a}}{T \cdot 10^{-3}}\right)
$$

Plotando o gráfico $\log g(\alpha) \times 1 / T$, obtêm-se a $E_{a}$ pela inclinação da reta e A pela intersecção da reta.

Já no método de van Krevelen et al, (1951) é utilizada a equação 6.

$$
\ln g(\alpha)=\ln B+\left[\frac{E_{a}}{R T_{\max }}+1\right] \ln T
$$

Onde,

$$
B=\frac{A}{\beta}\left[\frac{E_{a}}{R T_{\max }}+1\right]^{-1}\left[\frac{0,368}{T_{\max }}\right]^{E_{a} / R T_{\max }}
$$

Novamente, neste mecanismo é necessário plotar $\ln g(\alpha)$ versus $\ln T$ para obter os parâmetros cinéticos da reação.

\section{RESULTADOS}


A Figura 1 (a) apresenta a DTG e a figura 1 (b) apresenta TGA do MDF obtida nas diferentes taxas de aquecimento analisadas, no intervalo de temperatura compreendido entre 20 e $800{ }^{\circ} \mathrm{C}$ em atmosfera inerte.

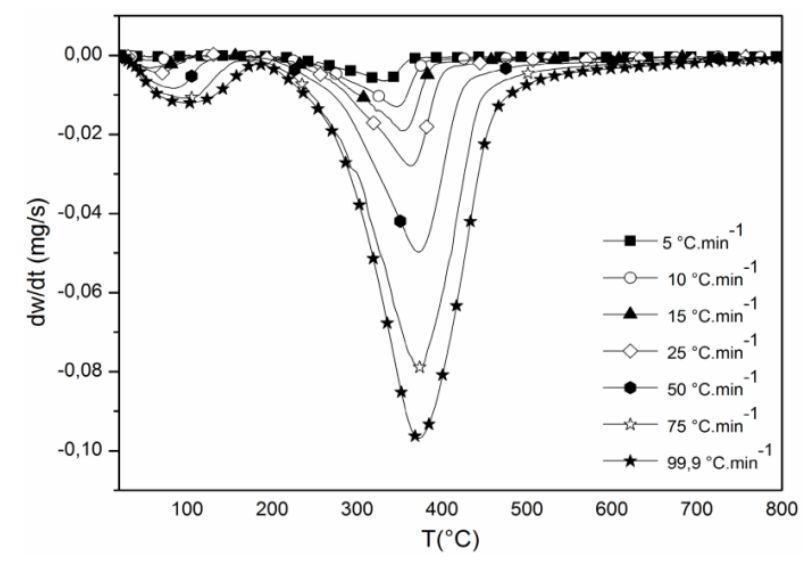

Figura 1 (a) - DTG do MDF.

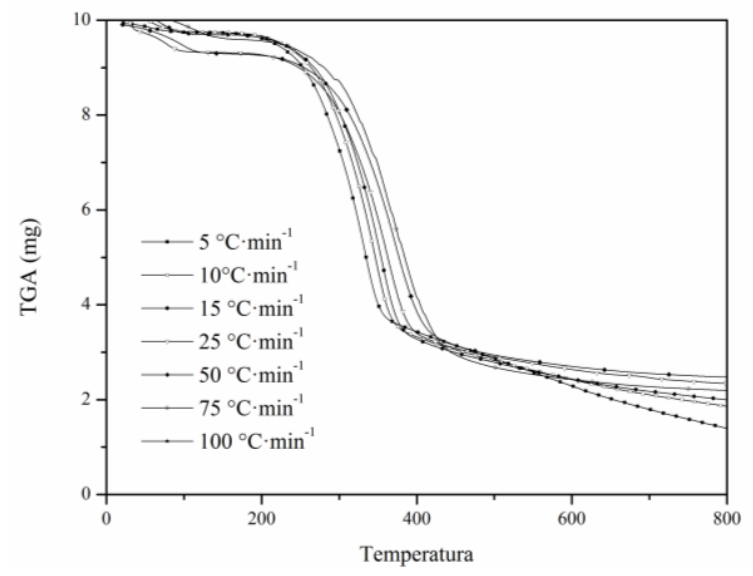

Figura 1 (b) - TGA do MDF.

Nas curvas de DTG é possível observar as temperaturas onde as taxas de reação são máximas. Com base na figura 1(a), foi definida a região de estudo, compreendida na faixa de 200 a $500{ }^{\circ} \mathrm{C}$, onde ocorre a principal perda de massa. É possível observar que quando há um aumento na velocidade de aquecimento a decomposição térmica ocorre a temperaturas mais elevadas. Conforme Bianchi (2010) a lignina presente na biomassa apresenta temperaturas de perda de massa mais elevadas que a celulose sendo considerada estável termicamente. Por esta razão, a máxima velocidade de decomposição está levemente deslocada para maiores temperaturas.

Na Figura 1- (b) o TGA apresenta três regiões distintas: a $1^{a}$ região está entre a temperatura ambiente e cerca de $100^{\circ} \mathrm{C}$, representando a volatilização da água e extrativos presentes na madeira; a $2^{\mathrm{a}}$ região, na qual ocorre a liberação da matéria volátil (entre $225-380^{\circ} \mathrm{C}$ ); e a $3^{\text {a }}$ região (temperaturas acima de $380^{\circ} \mathrm{C}$ ) em que ocorrem reações envolvendo produto carbonáceo (char) da reação da pirólise KERCHER e NAGLE (2001).

A Tabela 2 apresenta as temperaturas T(máx) onde a taxa de reação é máxima nas respectivas taxas de aquecimento para a amostra de MDF.

Tabela 2 - Temperaturas de Máximo

\begin{tabular}{cc}
\hline Taxa de aquecimento $\left({ }^{\mathbf{0}} \mathbf{C} \cdot \mathbf{m i n}^{-\mathbf{1}}\right)$ & $\boldsymbol{T}_{\max }$ \\
\hline 5 & 327.0 \\
10 & 340.1 \\
15 & 348.9 \\
25 & 358.2 \\
50 & 365.7 \\
75 & 368.3 \\
99.9 & 370.2 \\
\hline
\end{tabular}

A figura $2 \overline{\text { apresenta os diferentes mecanismos cinéticos testados na }}$ região estudada, através do método de Coats-Redfern para a taxa de aquecimento de $5^{\circ} \mathrm{C} \cdot \mathrm{min}^{-1}$. 


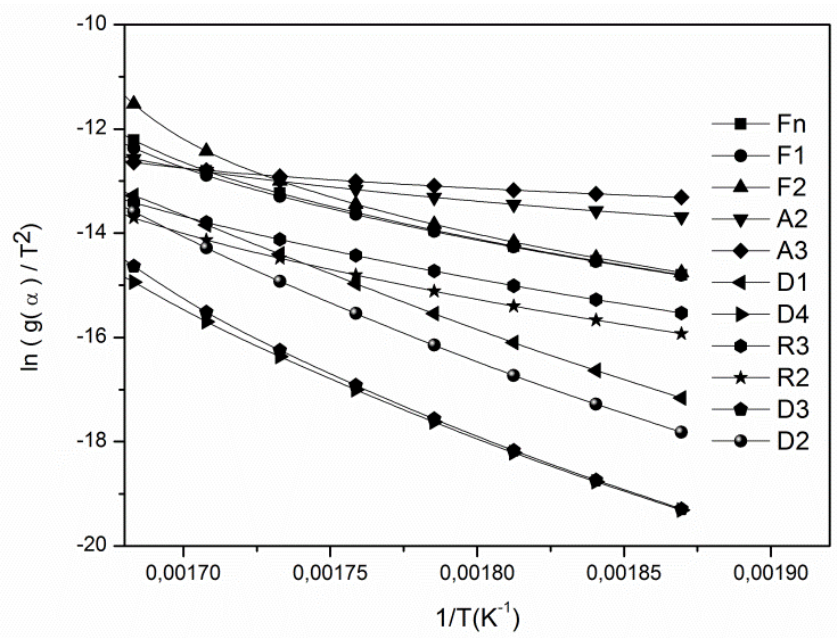

Figura 2 - Ajuste dos dados experimentais através dos diferentes mecanismos testados para o método de Coats-Redfern, na taxa de aquecimento de $5^{\circ} \mathrm{C} \cdot \mathrm{min}^{-1}$.

As demais taxas de aquecimento foram testadas através do mesmo método. A partir da Tabela 3 é possível identificar que o mecanismo D3 apresenta os coeficientes de correlação $\left(\mathrm{R}^{2}\right)$ mais próximos a unidade, indicando que a Difusão controla a cinética da reação de pirólise do MDF para todas as taxas de aquecimento.

Tabela 3 - Coeficientes de Correlação $\left(\mathrm{R}^{2}\right)$ para os mecanismos avaliados nas diferentes taxas de aquecimento

\begin{tabular}{lccccccc}
\hline Mechanism & $\boldsymbol{\beta ~ 5}$ & $\boldsymbol{\beta ~ 1 0}$ & $\boldsymbol{\beta ~ 1 5}$ & $\boldsymbol{\beta ~ 2 5}$ & $\boldsymbol{\beta ~ 5 0}$ & $\boldsymbol{\beta ~ 7 5}$ & $\boldsymbol{\beta} 100$ \\
\hline Orden n $(\boldsymbol{F n})$ & 0,9949 & 0,9710 & 0,9831 & 0,9788 & 0,9594 & 0,9986 & 0,9992 \\
First-order $(\boldsymbol{F 1})$ & 0,9979 & 0,9979 & 0,9950 & 0,9935 & 0,9938 & 0,9988 & 0,9990 \\
Second-order $(\boldsymbol{F} 2)$ & 0,9720 & 0,9674 & 0,9649 & 0,9589 & 0,9594 & 0,9828 & 0,9850 \\
Avrami-Erofeev $(\boldsymbol{A 2})$ & 0,9974 & 0,9960 & 0,9949 & 0,9992 & 0,9923 & 0,9830 & 0,9996 \\
Avrami-Erofeev $(\boldsymbol{A 3})$ & 0,9968 & 0,9949 & 0,9935 & 0,9898 & 0,9902 & 0,9974 & 0,9991 \\
One-Way transport $(\boldsymbol{D} 1)$ & 0,9895 & 0,9928 & 0,9934 & 0,9943 & 0,9922 & 0,9807 & 0,9798 \\
Valensi Equation $(\boldsymbol{D} 2)$ & 0,9961 & 0,9979 & 0,9985 & 0,9995 & 0,9996 & 0,9901 & 0,9898 \\
Jander Equation $(\boldsymbol{D} 3)$ & 0,9994 & 0,9996 & 0,9997 & 0,9997 & 0,9999 & 0,9997 & 0,9997 \\
Ginstling-Brounshtein $(\boldsymbol{D} 4)$ & 0,9979 & 0,9991 & 0,9993 & 0,9996 & 0,9990 & 0,9931 & 0,9931 \\
Contracting Sphere $(\boldsymbol{R} 2)$ & 0,9993 & 0,9996 & 0,9996 & 0,9997 & 0,9999 & 0,9930 & 0,9929 \\
Contracting Cylinder $(\boldsymbol{R 3})$ & 0,9982 & 0,9993 & 0,9995 & 0,9997 & 0,9993 & 0,9962 & 0,9965 \\
\hline
\end{tabular}

Uma vez, definido o mecanismo controlador da cinética de reação da pirólise, os dados das ordens de reação foram usados para determinar a energia de ativação $\left(E_{a}\right)$ e o fator pré-exponencial (A), utilizando os métodos de Coats-Redfern (CR), MacCallumTanner (MT) e Van Krevelen (VK) nas diferentes taxas de aquecimento avaliadas. Os resultados são fornecidos na Tabela 4.

Tabela 4 - Valores de energia de ativação e do fator pré-exponencial obtidos utilizando diferentes métodos de ajuste 


\begin{tabular}{lllllll}
\hline & CR & & MT & & VK & \\
\hline$\beta\left(\mathrm{K} \mathrm{min}^{-1}\right)$ & $\mathrm{E}_{\mathrm{a}}(\mathrm{kJ} / \mathrm{mol})$ & $\mathrm{A}\left(\mathrm{min}^{-1}\right)$ & $\mathrm{E}_{\mathrm{a}}(\mathrm{kJ} / \mathrm{mol})$ & $\mathrm{A}\left(\mathrm{min}^{-1}\right)$ & $\mathrm{E}_{\mathrm{a}}(\mathrm{kJ} / \mathrm{mol})$ & $\mathrm{A}\left(\mathrm{min}^{-1}\right)$ \\
\hline $\mathbf{5}$ & 139,184 & $2,53 \cdot 10^{10}$ & 141,050 & $3,46.10^{10}$ & 149,766 & $2,13.10^{11}$ \\
$\mathbf{1 0}$ & 140,839 & $3,95.10^{10}$ & 142,929 & $5,66.10^{10}$ & 152,378 & $3,861.10^{11}$ \\
$\mathbf{1 5}$ & 141,587 & $4,71.10^{10}$ & 143,747 & $5,84.10^{10}$ & 152,473 & $4,78.10^{11}$ \\
$\mathbf{2 5}$ & 137,862 & $2,46.10^{10}$ & 140,130 & $3,63.10^{10}$ & 150,041 & $2,55.10^{11}$ \\
$\mathbf{5 0}$ & 137,255 & $2,37.10^{10}$ & 139,741 & $3,66.10^{10}$ & 147,939 & $1,76.10^{11}$ \\
$\mathbf{7 5}$ & 137,123 & $2,42.10^{10}$ & 139,937 & $2,32.10^{10}$ & 150,580 & $1,63.10^{11}$ \\
$\mathbf{1 0 0}$ & 138,328 & $2,04.10^{10}$ & 141,149 & $4,10.10^{10}$ & 150,052 & $2,10.10^{11}$ \\
\hline
\end{tabular}

\section{CONCLUSÕES}

O estudo da cinética da reação de pirólise através da termogravimetria (TGA/DTG) para o MDF pode ser uma ferramenta útil para investigar as diferentes etapas de degradação térmica da biomassa.

A biomassa estudada neste trabalho mostra que a taxa de aquecimento no processo de pirólise, interfere na cinética da reação, pois tanto a energia de ativação quanto o fator pré-exponencial variaram com a variação da taxa de aquecimento. Os dados cinéticos em conjunção com os modelos utilizados mostram que o mecanismo predominante de decomposição térmica para o MDF é difusão.

Os experimentos em atmosfera inerte mostraram que a região onde ocorre a principal perda de massa do MDF acontece entre $200{ }^{\circ} \mathrm{C}$ e $500{ }^{\circ} \mathrm{C}$, a energia de ativação variou de 137 a $152{\mathrm{~kJ} . \mathrm{mol}^{-1}}^{-1}$ o fator pré-exponencial de $2.10^{10}$ a $6.10^{11} \mathrm{~min}^{-1}$.

\section{REFERÊNCIAS BIBLIOGRÁFICAS}

ABIPA - Associação Brasileira da Indústria de Painéis de Madeira. Disponível em: <http://www.abipa.org.br/produtosMDP.php/>. Acesso em 25 de outubro, 2012.

ABOULKAS, A.; HARFI, K. E.; BOUADILI, A. E.; NADIFIYINE, M.; BENCHANAA, M.; MOKHLISSE, A. Pyrolysis kinetics of olive residue/plastic mixtures by non-isothermal thermogravimetry. Fuel Processing Technology, n. 90, p. 722-728, 2009.

BIANCHI. O. et al. Avaliação da degradação não-isotérmica de madeira através de termogravimetria-TGA. Polímeros. Vol 2, n. especial, p. 395-400, 2010.

COATS, A. W.; REDFERN, J. P. Kinetic parameters from thermogravimetric data. Nature, n. 201, p. 68-69, 1964.

ELLA, E.S.; YUAN, G.; MAYS T. A simple kinetic analysis to determine the intrinsic reactivity of coal chars. Fuel 2005, 84, 1920-1925.

FRAGA, F.; NUNEZ, E. R. Activation energy for the epoxy system BADGE n Z 0/m- XDA obtained using data from thermogravimetric analysis. Journal of Applied Polymer Science, $n$. 80, p. 776-782, 2001.

GAN, Q.; ALLEN, S.J.; MATTHEWS, R, Activation of waste MDF sawdust charcoal and its reactive dye adsorption characteristics. Journal Management, v.24, p.841-848, 2004. 
GAO, X; WU, H. Combustion of volatiles produced in situ from the fast pyrolysis of woody biomass: direct evidence on its substantial contribution to submicrometer particle (PM1) emission. Energy Fuels 2011, 25, 4172-4181.

GHETTI, P.; RICCA, L.; ANGELINI, L. Thermal analysis of biomass and corresponding pyrolysis products. Fuel, v. 75, n. 5, p. 565-573, 1996.

HAGEDORN, M.M.; BOCKHORN, H.; KREBS, L.; MULLER U. A comparative kinetic study on the pyrolysis of three different wood species. J. Anal. Appl. Pyrolysis. V.68, 231-249, 2003.

JIANG, X.; ELLIS, N.; SHEN, D.; JIANG, J.; DAI, W.; ZHONG, Z. Thermogravimetry- FTIR analysis of pyrolytic lignin extracted from bio-oil. Chemical Engineering and Technology, v.35, p.827-833, 2010.

Kercher, A.K.; Nagle, D.C. TGA modeling of the thermal decomposition of CCA treated lumber waste. Wood Sci. Technol. v. 35. 325-341, 2001.

KIM, S.; KIM, Y.C. Using isothermal kinetic results to estimate the kinetic triplet of the Pyrolysis of high density polyethylene. Journal of Analytical and Applied Pyrolysis, n. 73, 117-121, 2005.

LUA, A. C.; SU, J. Isothermal and non-isothermal kinetics of Kapton® polyimide. Polymer Degradation and Stability, n. 91, p. 144-153, 2006.

MACCALLUM, J. R.; TANNER, J. The kinetics of thermogravimetry. European Polymer Journal, v. 6, p. 1033-1039, 1970.

MONTSERRAT, S.; MÁLEK, J.; COLOMER, P. Thermal degradation kinetics of epoxyanhydride resins: I. Influence of a silica filler. Termochimica Acta, v. 313, p. 83-95, 1998.

MUNIR, S.; DAOOD, S.S.; NIMMO, W.; CUNLIFFE, A.M.; GIBBS, B.M. Thermal analysis and devolatilization kinetics of cotton stalk, sugar cane bagasse and shea meal under nitrogen and air atmospheres. Bioresource Technology. v. 100, n. 3, 1413-1418, 2009.

PERONDI. D. et al, Thermal decomposition of polymeric resin [(c29h24n205)n]: kinetic parameters and mechanisms. Polymer Degradation and Stability, n 97, p.2110-2117, 2012.

REGLAND, K.W.; AERTS, D.J. Properties of wood for combustion analysis. Bioresour. Technol. 1991, 37, 161-168.

REGNIER, N.; GUIBE, C. Methodology for multistage degradation of polymide polymer. Polymer Degradation and Stability, n. 55, p. 165-172, 1997.

VAN KREVELEN, D. W.; VAN HEERDEN, C.; HUNTJENS, F. J. Physicochemical aspects of the pyrolysis of coal and related organic compounds. Fuel, n. 30, p. 253-258, 1951.

VLAEV, L.; NEDELCHEV, N.; GYUROVA, K.; ZAGORCHEVA, M. A comparative study of non-isothermal kinetics of decomposition of calcium oxalate monohydrate. Journal of Analytical and Applied Pyrolysis, n. 81, p. 253-262, 2008. 